\title{
Therapy of CF-Patients with Amitriptyline and Placebo - a Randomised, Double-Blind, Placebo-Controlled Phase IIb Multicenter, Cohort-Study
}

\author{
Lutz Nährlich ${ }^{\mathrm{a}, \mathrm{f}} \quad$ Jochen G. Mainz ${ }^{\mathrm{b}, \mathrm{f}}$ Constantin Adams ${ }^{c} \quad$ Corinna Engelc \\ Gloria Herrmannc Vanya Ichevac Josefine Lauer ${ }^{c}$ Caroline Deppisch ${ }^{c}$ \\ Andreas Wirth ${ }^{c}$ Katy Unger ${ }^{c}$ Ute Graepler-Mainkac Andreas Hector ${ }^{c}$ \\ Susanne Heyderc Martin Sternc ${ }^{c}$ Gerd Döring ${ }^{d}$ Erich Gulbins ${ }^{\mathrm{e}}$ \\ Joachim Riethmüller ${ }^{c}$
}

\begin{abstract}
${ }^{a}$ Department of Paediatrics, University Hospital Giessen, Giessen; 'Department of Paediatrics, University Hospital Jena, Jena; 'Department of Paediatrics, University Hospital Tuebingen, Tuebingen; dDepartment of Clinical Microbiology and Hygiene, University Hospital Tuebingen, Tuebingen; eDepartment of Molecular Biology, University of Duisburg-Essen, Essen; fthese authors contributed equally and share first authorship
\end{abstract}

\section{Key Words}

Cystic Fibrosis $•$ Amitriptyline $\bullet$ P. aeruginosa $\bullet$ Ceramide $\bullet$ CFTR

\begin{abstract}
Background/Aims: Several recent studies revealed an accumulation of ceramide in bronchial, tracheal and intestinal epithelial cells of mice and patients with cystic fibrosis (CF). Normalization of ceramide concentrations in lungs of CF mice employing the functional acid sphingomyelinase inhibitor amitriptyline also normalized mucociliary clearance, chronic inflammation and infection susceptibility to pulmonary $P$. aeruginosa in these mice. Methods: To test for a beneficial effect of amitriptyline in vivo, we performed a phase IIb randomised, double-blind, placebo-controlled study. Twenty-one CF patients were treated with $25 \mathrm{mg} / \mathrm{d}$ amitriptyline twice daily for 28 days. The placebo consisted of 19 patients and was also treated twice per day. The primary endpoint was the change in lung function in the intentionto-treat (ITT) population. Secondary endpoints were ceramide levels in epithelial cells and safety. Results: After treatment, forced expiratory volume in $1 \mathrm{sec}$ predicted $\left(\mathrm{FEV}_{1}\right)$ increased $6.3 \pm 11.5 \%(p=0.08)$ in the ITT population (36 of 40 CF patients) and $8.5 \pm 10 \%(p=0.013)$ in the per protocol (PP) population (29 of 40 patients). Ceramide levels decreased in nasal epithelial cells after amitriptyline treatment. Amitriptyline had no severe and only mild and mostly transient adverse effects, i.e. xerostomia and tiredness. Conclusion: Amitriptyline is safe in CF-patients, increases FEV $_{1}$ and reduces ceramide in lung cells of CF patients.
\end{abstract}

Copyright (C) 2013 S. Karger AG, Basel 
Nährlich et al.: Amitriptyline in CF

\section{Introduction}

Cystic fibrosis (CF) is the most common autosomal recessive disorder in western countries and caused by mutations in the cystic fibrosis transmembranous conductance regulator (CFTR) molecule [1-3]. The disease is characterized by chronic pulmonary inflammation, increased infection susceptibility and fibrosis [4, 5]. Chronic bacterial respiratory infections have the greatest impact on morbidity and mortality of the patients [5]. Most patients suffer from chronic infections with P. aeruginosa [6], the major opportunistic pathogen in CF. Mucoid P. aeruginosa grow in the bronchi of CF-patients, are surrounded by masses of dead neutrophils [6], and high doses of antibiotics are required for treatment of $C F$ lung disease, but even with aggressive treatment $P$. aeruginosa infections become chronic [7].

Several novel treatments including inhalation of Pulmozyme and application of CFTR correctors were introduced into the clinic within the last years [8]. However, inhalation of Pulmozyme, a DNase, does not prevent long-term decline of lung functions. CFTR correctors only promote the function of G551D mutants, while they do not promote function of CFTR in the vast majority of the patients [8].

Therefore, novel and improved treatments for CF are urgently required. Recent animal studies offer an attractive novel possibility to treat cystic fibrosis [9]: We demonstrated in mice genetically deficient for Cftr an accumulation of ceramide in tracheal and bronchial epithelial cells [9]. The results were recently independently confirmed and transferred to the human situation demonstrating accumulation of ceramide in bronchial epithelial cells of patients with cystic fibrosis [9-12]. Ceramide mediates increased death of bronchial epithelial cells resulting in a release of dead cells and finally DNA into the bronchial lumen [9]. The reduction of the mucociliary clearance by ceramide facilitates infections with $P$. aeruginosa [9]. In addition, ceramide induces chronic inflammation in CF lungs [9]. Genetic or pharmacological inhibition of acid sphingomyelinase (ASM), which releases ceramide, reduced pulmonary ceramide levels and normalized mucociliary clearance, inflammation and infection susceptibility [9]. Inhibition of the acid sphingomyelinase was achieved by treatment of CF mice with amitriptyline, a known functional blocker of the acid sphingmyelinase $[13,14]$.

These very encouraging animal data prompted us to test for a beneficial effect of amitriptyline in CF patients. Amitriptyline is well established in treatment of patients with major depression and clinical studies are warranted to assess tolerability and efficacy of amitriptyline for treatment of CF patients. We have previously assessed the therapeutic efficacy and safety of amitriptyline in a pilot study involving $4 \mathrm{CF}$ patients treated with amitriptyline or placebo demonstrating a significant increase in lung function after 2 weeks of treatment with amitriptyline. We subsequently performed a phase Ila study for safety, proofof-mechanism and dose-finding study involving $19 \mathrm{CF}$ patients [15]. This study revealed that amitriptyline is safe in CF patients, since only mild adverse effects were observed. Moreover, the per protocol (PP) analysis of this study demonstrated a 5\% increase of the forced expiratory volume in one second $\left(\mathrm{FEV}_{1}\right)$ after treatment with amitriptyline compared to the placebo group suggesting an improvement of lung functions by amitriptyline [15]. Although it is difficult to exactly determine the complex physiology and pathophysiology of the lung in CF patients, medical associations (European Medicines Agency, Food and Drug Administration, USA) suggested to use the $\mathrm{FEV}_{1}$, i.e. the maximal volume exhaled by patient within the $1^{\text {st }}$ second after maximal inhalation, as the most appropriate parameter to determine lung function.

Here we performed a phase IIb study on 40 CF-patients treated with $25 \mathrm{mg}$ amitriptyline twice daily. We demonstrate an increase of $\mathrm{FEV}_{1}$ in the intention-to-treat (ITT) and PP populations upon treatment of CF patients with amitriptyline for four weeks. The side effects of the treatment are minor and amitriptyline is well tolerated by CF patients. 
Nährlich et al.: Amitriptyline in CF

\section{Methods}

\section{Study design}

We performed a phase IIb study in a multicentric, randomised, double-blind, placebo-controlled, cohort study design in $40 \mathrm{CF}$ patients, attending the CF centre of the Justus-Liebig-University Giessen, the CF centre of the Children's University Hospital of Jena and the CF centre of the Children's University Hospital of Tübingen, Germany. The study was approved by the Institutional Review Board of the University Hospital Tübingen. All patients and parents of adolescent patients gave written informed consent and were insured for potential adverse effects. The phase IIb study was registered at EudraCT 2008-002673-13 and ClinicalTrials.gov, number NCT01309178.

\section{Patients}

Patients were included in the study based on the following criteria: cystic fibrosis was verified; patient was older than 14 years; patient's weight was more than $35 \mathrm{~kg}$; FEV ${ }_{1}$ was higher than $30 \%$ and lower than 90\%; patient was pulmonary colonized with bacteria; no acute pulmonary illness was present; C-reactive protein (CRP) was not elevated two fold ( $2 \mathrm{mg} / \mathrm{dl}$ ) over normal levels; lung function tests were possible and a full course of therapy was possible without any restrictions. Patients were excluded from the study based on the following criteria: $\mathrm{FEV}_{1}$ in baseline differed more than $10 \%$ from screening visit; CRP in baseline differed more than $50 \%$ from screening visit; glaucoma, seizures, heart insufficiency or major depression were present; intravenous antibiotic treatment was necessary in the last 4 weeks before inclusion visit; high dose steroid therapy was necessary; on/off-therapy of tobramycin was present in the last 2 weeks; involvement of the patient in another study; pregnancy and nursing mothers. Patients were randomized to amitriptyline capsules, administered orally at a dosage of $25 \mathrm{mg}$ amitriptyline per capsule, twice daily for 4 weeks, or the placebo (corn starch capsules) using the software StatsDirect 2.7.2 (StatsDirect Ltd; Altrincham, Cheshire, UK). During treatment period, patients' visits were at days -7, 0, 14 and 28. A follow up visit was initiated at day 36.

\section{Outcome}

The primary outcome was the difference of $\mathrm{FEV}_{1}$ predicted at day 28 to placebo in the ITT population measured by spirometry (Jaeger, Höchberg, Germany). Secondary endpoints were (i) absolute and relative $\mathrm{FEV}_{1}$ differences to baseline, (ii) ceramide concentrations in respiratory epithelial cells, (iii) bacterial counts, (iv) leukocyte counts, (v) concentrations of cytokines and (vi) DNA concentrations in sputum specimens. C-reactive protein (CRP) and the cytokines IL-1ß, IL-6, IL-8, IL-10 were determined in blood and sputum specimens using routine methods and the Bio-Plex 200 System (Bio-Rad Laboratories GmbH, München, Germany). C-reactive protein was measured as an additional inflammatory parameter in blood specimens using luminescence technology. Nasal epithelial cells were obtained from all patients for the semi-quantitative determination of ceramide using immunofluorescence [9]. To this end, the cells were stained with a Cy3-coupled monoclonal anti-ceramide antibody (Glycobiotech) and analysed by fluorescence microscopy. The relative amount of ceramide was determined by measuring the fluorescence intensity on a Leica fluorescence microscope DMIRE 2. The intensity of the staining was scored from weak to strong staining. DNA and number of bacterial pathogens were quantified electrophoretically [16] and by routine culturing on blood agar, respectively, at day 28.

For assessing safety of amitriptyline, blood pressure, heart rate, transaminases and creatinine values were measured at each visit. An electrocardiogram was taken at day 14. Adverse effects (AEs) and severe AEs (SAEs) were documented on patient sheets.

\section{Statistics}

Based on the results of our previous studies [15], we used the difference of relative $\mathrm{FEV}_{1}$ predicted between cases and controls after treatment of 28 days as primary endpoint.

Due to the fact, that $\mathrm{FEV}_{1}$ is measured as \%, geometric means were used to compare the results in the verum and the placebo group by student's t-test. Confirmative analysis was based on the ITT-population, with significance level $\alpha=0.05$. Secondary endpoints were $\mathrm{FEV}_{1}$ predicted absolute in the ITT and absolute and relative to baseline in the PP population and ceramide concentration. The latter is presented by frequencies and 95\%-confidence intervals of patients with decrease in ceramide concentration after 


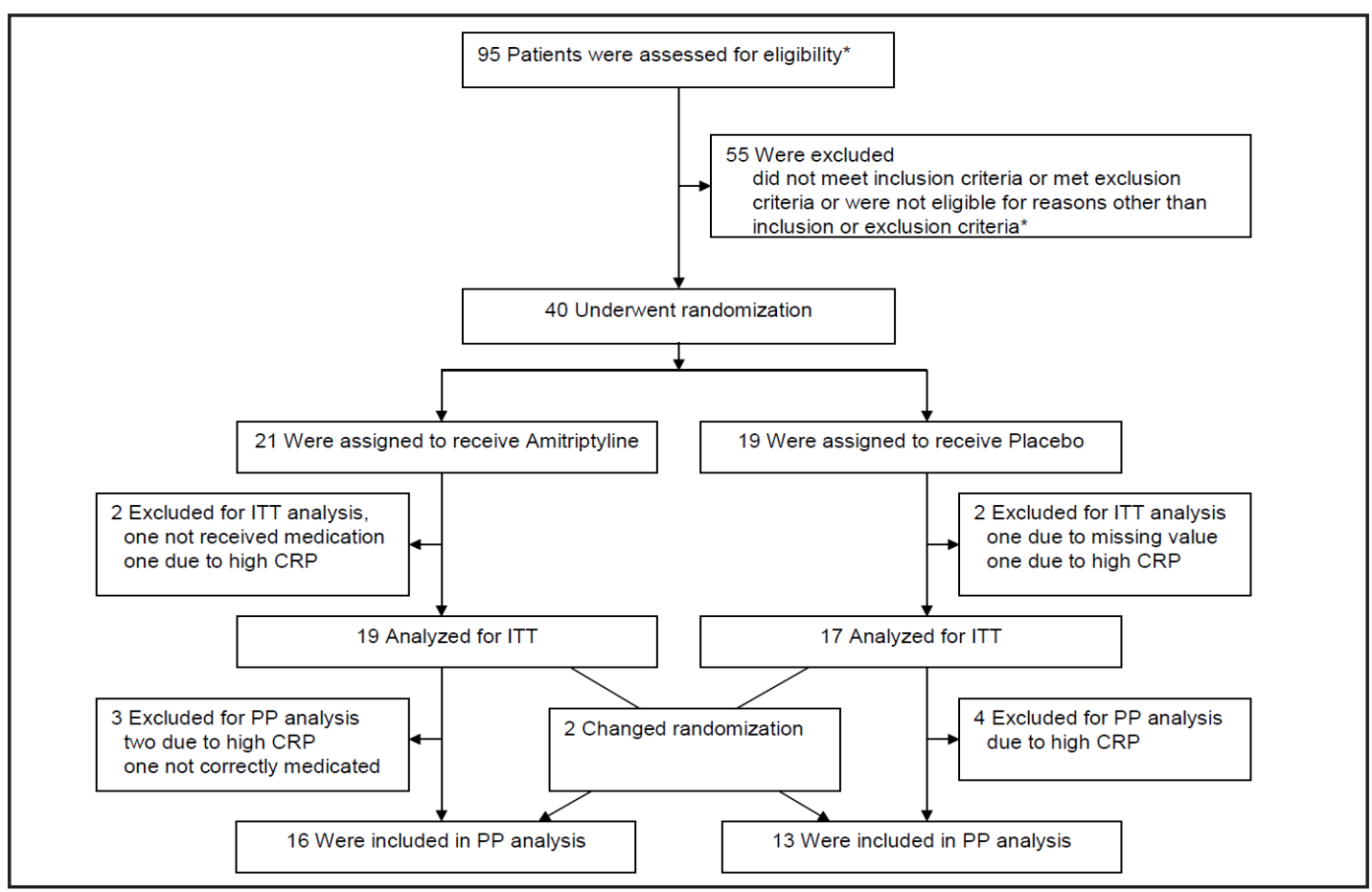

Fig. 1. CONSORT patient flow chart. Screening and randomization. *All CF patients older than 14 years admitted to the CF centres were assessed for eligibility. ITT: Intention to treat; PP: Per protocol. CRP: C-reactive protein.

treatment. Analysis of secondary endpoints or analysis based on the PP population was regarded as being descriptive.

The ITT analysis includes all patients who participated in the study, regardless any protocol violations. Even a patient, who was accidentally treated with the placebo instead with amitriptyline as happened in the present study, is included in the ITT analysis.

The PP population excluded all patients that did not take study medication according to the study protocol or showed high changes in CRP. Missing values were not replaced.

\section{Results}

Ninety-five patients were initially screened for the study. From the 40 adolescent and adult CF patients for this phase IIb study, 21 patients were randomized to receive amitriptyline and 19 patients to receive placebo treatment. Two patients were excluded from analysis due to high CRP and one patient due to missing lung function values at day 28 . The remaining 37 patients were included in the ITT analysis (19 females, 18 males; mean age: $25.5 \pm 8.4$ yrs) (Fig. 1). A further patient refused to take medication. Due to a mistake in randomisation, one patient in the amitriptyline group received placebo and one patient in the placebo group received amitriptyline. For PP analysis, seven patients were excluded during the study due to high CRP or not correctly medicated. Twentynine patients could be included in the perprotocol analysis (PP), 16 patients who received amitriptyline and 13 received placebo were analysed after correction of the randomisation mistake (Fig. 1). The demographic data of the patients (Table 1) reveal that both groups were similar with regard to gender, age, weight, lung function parameters, pancreatic sufficiency, and chronic bacterial infections.

After 28 days of amitriptyline treatment, lung functions of CF patients, measured as $\mathrm{FEV}_{1}$ predicted absolute and relative to baseline, improved in the ITT population to $0.6 \%$ and $0.2 \%$, respectively (Table 2 ). In contrast, placebo treatment resulted in a decrease of $\mathrm{FEV}_{1}$ in the ITT population of $3.8 \%$ and $6.1 \%$, respectively. The difference of $\mathrm{FEV}_{1}$ predicted absolute 


\begin{tabular}{lccc}
\hline & Amitriptyline & Placebo & \\
\hline Characteristic & & $(n=19)$ & $p$ value \\
Female Gender (\%) & $9(42)$ & $10(56)$ & 0.44 \\
Age (years) a & $25.1 \pm 8.2$ & $25.8 \pm 8.9$ & 0.32 \\
Weight (kg) & $56.9 \pm 8.9$ & $53.0 \pm 6.5$ & 0.23 \\
FEV $\%^{*}$ & $65.0 \pm 15.6$ & $58.4 \pm 17.7$ & 0.24 \\
FVC \% & $81.4 \pm 13.3$ & $75.5 \pm 12.8$ & 0.19 \\
MEF $25-75 \% *$ & $30.6 \pm 23.4$ & 0.51 \\
Pancreas insufficiency (\%) & $35.4 \pm 17.7$ & 0 & n.a. \\
P. aeruginosa, non mucoid (mean logcfu) & $2(10.5)$ & $8(6.7)$ & 0.44 \\
P. aeruginosa, mucoid (mean logcfu) & $8(7.5)$ & $7(6.9)$ & 0.5 \\
St. aureus (mean logcfu) & $7(6.4)$ & $10(6.6)$ & 0.5 \\
MRSA & $10(6.3)$ & 0 & n.a. \\
Stenotrophomonas maltophilia (mean logcfu) & 1 & $2(7.7)$ & 0.06 \\
Aspergillus fumigatus & $6(5.8)$ & 3 & 0.5 \\
Candida albicans & 3 & 3 & 0.09 \\
E. coli & 7 & 1 & n.a. \\
others & 1 & 3 & n.a. \\
\hline a Values are means \pm SD; MRSA: methicillin resistant Staphylococcus aureus & &
\end{tabular}

Table 1. Demographic data and baseline characteristics in cystic fibrosis patients treated in the phase IIb amitriptyline study. Presented are the characteristics of all included individuals, i.e. gender, age, weight, the forced expiratory volume in the $1^{\text {st }}$ second $\left(\mathrm{FEV}_{1}\right)$, the forced vital capacity (FVC), the expiratory flow at 25 , 50 and $75 \%$ of the forced vital capacity $\left(\mathrm{MEF}_{25-75}\right)$, the percentage of patients with pancreas insufficiency, the logarithmic average of colony forming units (logcfu) of $P$. aeruginosa, $S$. aureus, multiple resistant $S$. aureus, S. maltophilia, A. fumigatus, Candida albicans, E. coli and other, not further specified bacteria in the lung.

\begin{tabular}{ccccccc}
\hline Population & Treatment group & $\begin{array}{c}\text { Number of } \\
\text { patients }\end{array}$ & \multicolumn{3}{c}{ FEV (\% predicted) } \\
& & $\begin{array}{c}\text { Absolute to } \\
\text { baseline }\end{array}$ & $p$ value & $\begin{array}{c}\text { Relative to } \\
\text { baseline }\end{array}$ & $p$ value \\
\hline ITT & Amitriptyline & 19 & $+0.6 \pm 5.7^{*}$ & 0.034 & $+0.2 \pm 11.4$ & 0.08 \\
& Placebo & 17 & $-3.8 \pm 6.9$ & & $-6.1 \pm 11.5$ & \\
PP & Amitriptyline & 16 & $+2.2 \pm 5.2$ & 0.013 & $+3.6 \pm 7.9$ & 0.013 \\
& Placebo & 13 & $-2.7 \pm 5.0$ & & $-4.9 \pm 10.1$ & \\
* values represent geometric means \pm standard deviations. Student's t-test was used & & \\
\hline
\end{tabular}

Table 2. Efficacy of amitriptyline in patients with cystic fibrosis. Lung function was determined in the Intention to treat (ITT) and the per protocol (PP) population as forced expiratory volume in one second $\left(\mathrm{FEV}_{1}\right)$ after 28 days of amitriptyline or placebo treatment in a phase IIb cohort study. The number (n) of patients in the different groups is given. Values represent geometric means \pm standard deviations. Significant difference of $\mathrm{FEV}_{1}$ between placebo and amitriptyline-treated groups are indicated by an asterisk $\left(^{*}\right), p \leq 0.05$, Student's t-test.

to baseline between amitriptyline and placebo was statistically significant $(p=0.03)$, while the primary endpoint, i.e., $\mathrm{FEV}_{1}$ predicted relative to baseline, was not significant $(\mathrm{p}=0.08)$ (Table 2). Five of 19 patients in the placebo group showed a $10 \%$ or more decline of $\mathrm{FEV}_{1}$ after 28 days of therapy because of lower respiratory tract infections, whereas no patient with such a high decline was found in the amitriptyline group.

The PP analysis revealed a significant increase of the primary endpoint, i.e. both $\mathrm{FEV}_{1}$ predicted absolute and relative to baseline improved significantly by $4.9 \%(p=0.013)$ and $8.5 \%(\mathrm{p}=0.013)$, respectively (Table 2).

The PP analysis also demonstrated a decrease of the secondary endpoint ceramidelevels in airway epithelial cells after 28 days of treatment with amitriptyline. The relative fluorescence intensities for ceramide in epithelial cells were scored by a blinded investigator with weak (1), mediate (2), strong (3) and very strong (4). The relative fluorescence intensity 
Nährlich et al.: Amitriptyline in CF

\begin{tabular}{|c|c|c|c|}
\hline & Amitriptyline & Placebo & \\
\hline Adverse effects* & $(n=45)$ & $(n=23)$ & $p$ value ${ }^{* *}$ \\
\hline Xerostomia (\%) & $14(82)$ & $3(18)$ & 0.053 \\
\hline transient & 9 & 3 & \\
\hline Tiredness (\%) & $\begin{array}{c}17(71) \\
12\end{array}$ & $\begin{array}{c}7(29) \\
6\end{array}$ & 0.28 \\
\hline Exacerbation & 0 & 1 & 0.08 \\
\hline Common cold (\%) & $11(65)$ & $6(35)$ & 0.44 \\
\hline Headache (\%) & $1(33)$ & $2(67)$ & 0.11 \\
\hline Gastroenteritis (\%) & $1(33)$ & $2(67)$ & 0.11 \\
\hline Pruritus & 0 & 1 & 0.08 \\
\hline Dizziness & 1 & 0 & 0.24 \\
\hline Sleep problems & 0 & 1 & 0.08 \\
\hline
\end{tabular}

Table 3. Safety of amitriptyline in CF patients during the phase IIb amitriptyline study*. Presented are the adverse effects observed in the present study in the amitripytline and the placebo groups. Sixty-eight adverse effects were observed in 30 out of 40 patients. *: The safety of amitriptyline was assessed after each treatment course of 28 day; **: Student's t-test was used for all statistical determinations, $\mathrm{p} \leq 0.05$.

for ceramide in the PP population decreased in 9 of 14 patients (64\%; 95\%-confidence interval (CI) 35-87\%) in the amitriptyline group. However, we also observed a change of ceramide intensities in 6 of 13 patients (46\%; 95\%-CI 19-75\%) of the placebo group after 28 days of treatment. The other secondary endpoints, i.e., bacterial counts, leukocyte counts, concentrations of cytokines and DNA concentrations in sputum specimens of CF patients were not significantly different between the amitriptyline and the placebo group (data not shown).

Amitriptyline was well-received in the patient group and no SAE was observed in the amitriptyline-treated group. One SAE was recorded at the end of the 28 day course in the placebo group. Sixty-eight AEs were observed in 30 out of 40 patients. Thirty-one AEs were related or possibly related to the medication. The incidence of two well-known AEs of amitriptyline, xerostomia and tiredness $[14,15]$, were different between the placebo and the amitriptyline group. However, the effect was mostly transient (Table 3). None of the other typical adverse effects of amitriptyline, i.e., vertigo, arterial hypotonia, long QT syndrome, tremor, dyskinesia, obstipation, dysuria or glaukoma were observed in our study, probably because of the low dose administered. Taken together, our data demonstrate a positive trend of amitriptyline to increase lung function and decrease ceramide levels in CF patients.

\section{Discussion}

In the present study, we tested whether amitriptyline improves lung functions in patients with CF. Since data from our phase IIa study [15] indicated that amitriptyline therapy using 25 to $75 \mathrm{mg}$ given twice daily for 14 days is safe and increases $\mathrm{FEV}_{1}$, we performed the present phase IIb trial to evaluate the effect of amitriptyline on $\mathrm{FEV}_{1}$ in a ITT and PP protocol. Further, the present study served to define the number of patients required for a phase III study using ITT population.

The present phase IIb study demonstrates a significant increase of the lung function of CF-patients after treatment with amitriptyline in the PP population. The positive effect of amitriptyline on lung function is supported by the finding that ceramide levels determined in airway epithelial cells decreased under amitriptyline therapy. However, future studies require a more quantitative analysis of ceramide, for instance analysis of the samples by mass spectrometry or ceramide kinase assays. Further, it might be better to use nasal brushes to obtain clearly-defined cell populations instead of sputum.

The study also demonstrates that amitriptyline, a well-established agent in treatment of major depression in adults [14], is safe at a dose of $2 \times 25 \mathrm{mg} /$ day in all CF patients when 
given orally for a period up to four weeks. No severe adverse drug reactions were reported, while typical AEs related to amitriptyline such as xerostomia and tiredness occurred. Importantly, AEs were mostly transitory and occurred only during the first days of therapy. Although CF patients seem to have an altered metabolism in general [17], the frequency and nature of side effects related to amitriptyline in those patients did not differ from reported adverse drug reactions of the drug in non-CF patients.

A further concern for the use of amitriptyline in CF patients infected chronically with $P$. aeruginosa or other bacterial pathogens, is the fact that amitriptyline might prevent the acute increase of ceramide levels in the respiratory tract observed after $P$. aeruginosa infections, which seems to be part of the host defence [18]. However, amitriptyline-treatment normalises, but does not reduce ceramide levels in cystic fibrosis lungs leaving the local response to $P$. aeruginosa intact $[9,18]$. Accordingly, the number of acute exacerbations did not increase significantly in the amitriptyline group compared to controls demonstrating again the safety of the applied amitriptyline dose in the CF patient study group. The observation that 5 of 19 patients in the placebo group showed a more than $10 \%$-decline of $\mathrm{FEV}_{1}$ after 28 days observation contrasts to a decline of 1 - $2 \%$ per year reported by other studies [5] and might be caused by the relatively short observation period in the present study. However, such a high decline was not seen in the amitriptyline-treatment group in the present study treated in parallel and this finding may support the notion of a beneficial effect of amitriptyline on lung functions.

Ceramide might have several actions in the lung. First, increased ceramide concentrations in bronchial epithelial cells were shown to trigger cell death and to mediate a release of DNA into the airways [9]. DNA reduces the mucociliary clearance and facilitates infections with $P$. aeruginosa [9]. Further, ceramide was shown to increase the pulmonary concentrations of pro-inflammatory mediators and trigger inflammation. The molecular mechanisms of this effect are presently unknown. All of these changes are prevented by normalization of pulmonary ceramide concentrations. Thus, the effects of amitriptyline on $\mathrm{FEV}_{1}$ observed in the present study might be caused by a combination of normalization of epithelial cell death and mucociliary clearance and a reduction of pulmonary inflammation.

In summary, the present findings demonstrate the safety and the efficacy of a treatment of CF patients with a low dose of amitriptyline and justify the continuation of the development of amitriptyline as a new strategy to treat CF patients in a future phase III study. It should be pointed out that an increase of $5-8 \%$ of lung function $\left(\mathrm{FEV}_{1}\right)$ is comparable to the beneficial effects of other drugs commonly used to treat cystic fibrosis. Furthermore, the potential of drugs that target the acid sphingomyelinase in cystic fibrosis might be increased by more efficient inhibition of the acid sphingomyelinase and/or local pulmonary application of higher doses without systemic effects.

\section{Conflict of interest}

We declare that we have no conflict of interest.

\section{Acknowledgements}

The Phase IIb trial was supported by a financial grant of the AKF-program of the University of Tübingen and a local German Cystic Fibrosis Association. E.G. was supported by DFG grant GU 335/16-2.

The sponsors had no role in study design, collection, analysis, and interpretation of data, writing of the report and in the decision to submit the paper for publication.

JR and EG planned the project. LN, JGM and JR conducted the study. JR conceived the project, was principal investigator, and co-wrote the paper with EG. CE contributed to the project design and analysed the data. GH, VI, CD, AW and KU analysed data and tested all 
patient samples in the various assays. EG, JL and CA tested ceramide levels. UGM, AH, SH and MS screened patients. GD reviewed the manuscript.

\section{References}

1 Rommens JM, Iannuzzi MC, Kerem B, Drumm ML, Melmer G, Dean M, Rozmahel R, Cole JL, Kennedy D, Hidaka N, et al.: Identification of the cystic fibrosis gene: chromosome walking and jumping. Science 1989;245:1059-1065.

- Riordan JR, Rommens JM, Kerem B, Alon N, Rozmahel R, Grzelczak Z, Zielenski J, Lok S, Plavsic N, Chou JL, et al.: Identification of the cystic fibrosis gene: cloning and characterization of complementary DNA. Science 1989;245:1066-1073.

3 Kerem B, Rommens JM, Buchanan JA, Markiewicz D, Cox TK, Chakravarti A, Buchwald M, Tsui LC: Identification of the cystic fibrosis gene: genetic analysis. Science 1989;245:1073-1080.

4 Ratjen FA: Cystic fibrosis: pathogenesis and future treatment strategies. Respir Care 2009;54:595-605.

5 CF Foundation, Patient Registry Annual Report 2004, Bethesda, Maryland, USA, 2005. http://www.cff.org/ UploadedFiles/publications/files/2006

6 Worlitzsch D, Tarran R, Ulrich M, Schwab U, Cekici A, Meyer KC, Birrer P, Bellon G, Berger J, Weiss T, Botzenhart K, Yankaskas JR, Randell S, Boucher RC, Döring G: Reduced oxygen concentrations in airway mucus contribute to the early and late pathogenesis of Pseudomonas aeruginosa CF airways infection. J Clin Invest 2002;109:317-325.

7 Döring G, Hoiby N, for the Consensus Study Group: Early intervention and prevention of lung disease in cystic fibrosis: a European consensus. J Cyst Fibros 2004;3:67-91.

-8 Ramsey BW, Davies J, McElvaney NG, Tullies E, Bell SC, Dřevínek P, Griese M, McKone EF, Wainwright CE, Konstan MW, Moss R, Ratjen F, Sermet-Gaudelus I, Rowe SM, Dong Q Rodriguez S, Yen K, Ordoñez C, Elborn JS, VX08-770-102 Study Group: A CFTR potentiator in patients with cystic fibrosis and the G551D mutation. New Engl J Med 2011;365:1663-1672.

-9 Teichgräber V, Ulrich M, Endlich N, Riethmüller J, Wilker B, De Oliveira-Munding CC, van Heeckeren AM, Barr ML, von Kürthy G, Schmid KW, Weller M, Tümmler B, Lang F, Grassmé H, Döring G, Gulbins E: Ceramide accumulation mediates inflammation, cell death and infection susceptibility in cystic fibrosis. Nat Med 2008;14:382-391.

10 Brodlie M, McKean MC, Johnson GE, Gray J, Fisher AJ, Corris PA, Lordan JL, Ward C: Ceramide is increased in the lower airway epithelium of people with advanced cystic fibrosis lung disease. Am J Respir Crit Care Med 2010;182:369-375.

11 Bodas M, Min T, Mazur S, Vij N: Critical modifier role of membrane-cystic fibrosis transmembrane conductance regulator-dependent ceramide signaling in lung injury and emphysema. J Immunol 2010;186:602-613.

12 Bodas M, Min T, Vij N: Critical role of CFTR-dependent lipid rafts in cigarette smoke-induced lung epithelial injury. Am J Physiol Lung Cell Mol Physiol 2011;300:L811-820.

13 Hurwitz R, Ferlinz K, Sandhoff K: The tricyclic antidepressants desipramine causes proteolytic degradation of lysosomal sphingomyelinase in human fibroblasts. Biol Chem Hoppe Seyler 1994;375:447-450.

$\checkmark 14$ Kornhuber J, Tripal P, Reichel M, Terfloth L, Bleich S, Wiltfang J, Gulbins E: Identification of new functional inhibitors of acid sphingomyelinase using a structure-property-activity relation model. J Med Chem 2008;51:219-237.

15 Riethmueller J, Anthonysamy J, Serra E, Schwab M, Döring G, Gulbins E: Therapeutic efficacy and safety of amitriptyline in patients with cystic fibrosis. Cell Physiol Biochem 2009;24:65-72.

16 Riethmueller J, Vonthein R, Borth-Bruhns T, Grassmé H, Eyrich M, Schilbach K, Stern M, Gulbins E: DNA quantification and fragmentation in sputum after inhalation of recombinant human deoxyribonuclease. Cell Physiol Biochem 2008;22:347-352.

17 Parker AC, Pritchard P, Preston T, Smyth RL, Choonara I: Enhanced drug metabolism in young children with cystic fibrosis. Arch Dis Childhood 1997;77:239-241.

18 Grassmé H, Jendrossek V, Riehle A, von Kürthy G, Berger J, Schwarz H, Weller M, Kolesnick R, Gulbins E: Host defense against Pseudomonas aeruginosa requires ceramide-rich membrane rafts. Nat Med 2003;9:322-330. 